\title{
Kernos
}

Revue internationale et pluridisciplinaire de religion grecque antique

$14 \mid 2001$

Varia

\section{Éléments orphiques chez Homère}

\section{Gregory Nagy}

\section{(2) OpenEdition}

Journals

\section{Édition électronique}

URL : http://journals.openedition.org/kernos/760

DOI : $10.4000 /$ kernos.760

ISSN : 2034-7871

\section{Éditeur}

Centre international d'étude de la religion grecque antique

\section{Édition imprimée}

Date de publication : 1 janvier 2001

Pagination : 1-9

ISSN : 0776-3824

Référence électronique

Gregory Nagy, "Éléments orphiques chez Homère », Kernos [En ligne], 14 | 2001, mis en ligne le 14 avril 2011, consulté le 02 mai 2019. URL : http://journals.openedition.org/kernos/760 ; DOI : 10.4000/ kernos.760 


\section{Éléments orphiques chez Homère}

Cette recherche est guidée par deux métaphores appliquées au texte d'Homère par les érudits de la bibliothèque d'Alexandrie et par ceux de la bibliothèque de Pergame. Dans le cas d'Alexandrie, la métaphore est celle du « corpus ", dans le cas de Pergame, il s'agit du « cosmos ». De part et d'autre, l'idée directrice est que l'édition d'Homère doit être entière ou complète. Mon argument principal est que ces deux métaphores, le « corpus » d'une part, et le « cosmos » de l'autre, sont significatives de l'attitude éditoriale d'Alexandrie et de Pergame touchant aux éléments orphiques chez Homère. Par « orphique », j'entends ici simplement tout trait que ces éditeurs peuvent avoir considéré comme relevant du personnage d'Orphée.

Un élément fondamental du prestige académique de la bibliothèque de Pergame était l'édition d'Homère par le bibliothécaire en chef, Cratès de Mallos, qui était un contemporain d'Aristarque de Samothrace, directeur de la bibliothèque d'Alexandrie vers le milieu du II $^{\mathrm{e}}$ siècle av. J.-C. Cratès appliquait

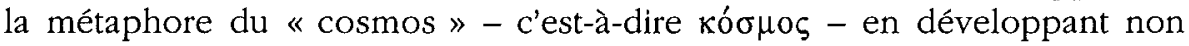
seulement les fondements philosophiques, mais aussi philologiques, de son édition de l'Tliade et de l'Odyssée homériques. Une telle métaphore était à même de donner un sens à l'extension maximale du texte d'Homère comme l'éditait Cratès à Pergame ${ }^{1}$. C'est en contraste avec l'« expansibilité » de l'Homère pergaménien - cet «Homerus auctus » - que se situait la compression du texte d'Homère dans l'édition d'Aristarque à Alexandrie ${ }^{2}$. En dépit d'un tel contraste, comme nous allons le voir, la métaphore du « cosmos » peut s'appliquer tout autant à ce texte homérique.

Notre première impression à propos de la métaphore du « corpus », par contre, était qu'elle s'appliquait uniquement au texte d'Homère tel qu'édité à Alexandrie. Ainsi qu'on le montrera plus avant, cette métaphore est évidente dans l'emploi du terme sôma en référence, non seulement au corps momifié d'Alexandre le Grand, qui se trouvait à côté de la bibliothèque d'Alexandrie,

1 Pour l'arrière-plan, voir G. NAGY, "The Library of Pergamon as a Classical Model", in H. Koester (éd.), Pergamon: Citadel of the Gods, Harrisburg PA, Trinity Press International, 1998, p. 185-232. Cet article est une version élargie de la présente étude. Ce qui est neuf est l'attention portée ici aux éléments orphiques chez Homère.

2 Ibid. 
mais aussi, par métonymie, à toute la collection de la bibliothèque ${ }^{3}$. Toutefois, comme on le montrera, cette première impression n'est pas pertinente, car la métaphore du « corpus » s'applique aussi au texte homérique édité à Pergame.

Quoi qu'il en soit, commençons par l'Alexandrie ptolémaïque. Dans ce cas, la métaphore du « corpus » reflète tout d'abord l'héritage du mythe et du rituel égyptiens. D'un point. de vue égyptien, la conservation d'un " corps » de livres est parallèle à celle du corps de pharaon par la momification, qui est la dimension rituelle de l'immortalisation (la momification dans le rituel est analogue à l'immortalisaton dans le mythe). À l'instar du corps de pharaon, le « corps » de livres est préservé en vue de sa conservation. En d'autres termes, pour la bibliothèque d'Alexandrie, l'idée de "corpus » dépend, au moins en partie, de conceptions religieuses égyptiennes touchant au corps momifié du roi-pharaon comme réincarnation exemplaire du dieu Osiris dont un récit sacré rapporte que le corps fut démembré ou dispersé pour être finalement rassemblé comme modèle de conservation éternelle ${ }^{4}$.

Dans le cas de l'Alexandrie ptolémaïque, la métaphore du «corpus » est explicite. Dans la description de la bibliothèque d'Alexandrie que livre Strabon (XVII, 1, 8 [C793-4]), où le mot-clé exedra indique que le référent est effectivement la bibliothèque, on lit que la tombe d'Alexandre était appelée le Sôma, « Corps » par excellence ${ }^{5}$. La bibliothèque d'Alexandrie est envisagée comme attenant au corps du roi, à l'image d'un corpus total. Le corpus de livres est coextensif du corps du roi, qui est le «corps politique ${ }^{6}$. La proximité sémantique, et même physique, de la bibliothèque et du corps du roi est, selon moi, une conception traditionnelle inhérente à l'idée même de bibliothèque ${ }^{7}$. Toutefois, un tel concept ne peut être considéré seulement en

3 Ibid.

4 Ibid.

5 La tradition manuscrite de Strabon porte $\Sigma \hat{\omega} \mu \alpha$, signifiant littéralement « corps», que les éditeurs modernes corrigent classiquement en $\Sigma \hat{\eta} \mu \alpha$, signifiant « tombeau ». Je propose de faire de $\Sigma \hat{\omega} \mu \alpha$ la lectio difficilior. Je reviens aujourd'hui sur ma lecture $\Sigma \hat{\eta} \mu \alpha$, dans G. NAgy, Pindar's Homer: The Lyric Possession of an Epic Past, Baltimore, Johns Hopkins Univ. Press, 1990, p. 272, n. 110. Dans le Roman d'Alexandre (II, 34, 5), il apparaît clairement que le taphos, "la tombe », d'Alexandre était appelée le $\sigma \hat{\omega} \mu \alpha$ 'A $A \varepsilon \xi \alpha^{\alpha} v \delta \rho o v$, "le tombeau d'Alexandre ". La métonymie qui fait du corps (sôma) par excellence le tombeau (sêma) est renforcée par le parallélisme phonétique de sôma / sêma.

6 Sur le symbolisme traditionnel du corps du roi comme incarnation du corps politique, $c f$. NagY, Pindar's Homer, p. 158, 177, 188, 258, 272

7 Cı́ć́ron utilise le mot grec sôma pour désigner un « corpus » de livres : Lettres à Atticus II, 1, 3; comparer l'utilisation du mot corpus dans la lettre à Lucceius (Ad Familiares $\mathrm{V}, 12,4)$. On peut également noter l'expression tò $\sigma \hat{\omega} \mu \alpha \tau \hat{\omega} v \gamma \rho \alpha \varphi \hat{\omega} v$, « le corpus

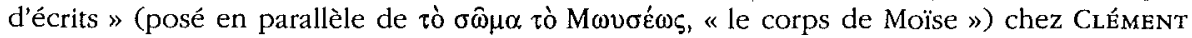
D'Alexandrie, Stromates VI, 132, 2-3. Pour ces exemples et d'autres plus anciens (Philon, De la vie contemplative, 78 et L'assomption de Moise, 93), voir Annewies vAN DEN HOEK, 
des termes égyptiens. La métonymie par laquelle le corps (sôma) par excellence est la tombe (sêma), renforcée par le parallélisme phonétique de sôma / sêma, ne peut être considérée seulement dans la perspective égyptienne : le jeu délibéré sur sôma / sêma est également « orphique », comme l'atteste la formulation explicite de Platon dans le Cratyle $(400 \mathrm{c})$.

À l'instar du « corpus », la métaphore « cosmos » reflète en partie l'héritage du mythe et du rite égyptiens, et plus spécialement ptolémaïques. Le texte alexandrin d'Homère peut être considéré comme un « cosmos » dans les termes du «mythe de fondation» ptolémaiqque d'Alexandrie : selon ce mythe, la cité fut fondée par Alexandre le Grand parce qu'Homère en personne lui était apparu en rêve et lui avait donné des ordres en ce sens (Plutarque, Vie d'Alexandre, 26, 5). À cause d'Homère, Alexandrie devient le centre du kosmos, gouverné par Alexandre en tant que souverain absolu, c'est-à-dire en kosmokratôr, selon les termes ptolémaïques ${ }^{8}$.

Les associations égyptiennes de ces deux métaphores ne doivent pas en faire oublier les associations non égyptiennes. Ces associations non égyptiennes des métaphores de « corpus » et de « cosmos » peuvent être liées à ce que j'ai appelé plus haut les éléments orphiques chez Homère. Dans les traditions poétiques de l'Athènes du $\mathrm{v}^{\mathrm{e}}-\mathrm{Iv}^{\mathrm{e}}$ siècle, le personnage d'Orphée et l'autre figure poétique exemplaire qu'était Musée sont conventionnellement dépeints comme antérieurs à Hésiode et Homère, ainsi que l'atteste le locus classicus des Nuées d'Aristophane (1032-1035). Parmi les autres témoignages importants figurent Hippias (86 B 6 D-K), Platon (Apologie, 41a; Rép., 363a, $377 \mathrm{~d}, 612 \mathrm{~b})$, Éphore $(70 \mathrm{~F} 101 \mathrm{~J})^{9}$. À cela, il faut ajouter l'idée traditionnelle qu'Homère était un descendant d'Orphée (Phérécyde, $3 \mathrm{~F} 167 \mathrm{~J}$; Hellanicos, 4 F $5 \mathrm{~J}$ ) ou de Musée (Gorgias, 82 B 25 D-K; Damastes, 5 F $11 \mathrm{~J}$ ).

Au contraire, Hérodote (II, 53, 3) s'oppose au point de vue qui place ces poètes avant Homère et Hésiode. Le contexte atteste clairement qu'Hérodote fait d'Homère et d'Hésiode les poètes les plus anciens. Ce ne peut être une coïncidence, à mon avis, que le point de vue relativement peu conventionnel d'Hérodote prenne place dans le contexte plus large de son discours global sur l'Égypte. Ce ne peut être une coïncidence non plus qu'Hérodote donne à Orphée et aux traditions orphiques une origine égyptienne, par le biais de

\footnotetext{
"The Concept of $\sigma \hat{\omega} \mu \alpha \tau \hat{\omega} v$ $\gamma \rho \alpha \varphi \hat{\omega} v$ in Alexandrian Theology", Studia Patristica 19 (1989), p. 250-254.

8 Sur l'idée de "mythe de fondation » en référence à la Vie d'Alexandre de Plutarque $(26,5)$, voir G. Nagr, Poetry as Performance: Homer and Beyond, Cambridge Univ. Press, 1991, p. 201-203. Sur Alexandre le Grand comme kosmokratôr (Roman d'Alexandre $1,7,3 ; 17,4$ ), voir Martha PAYNE, "Alexander the Great: Myth, the Polis, and Afterward", in D.C. Pozzi, J.M. Wickersham (éds), Myth and the Polis, Ithaca, Cornell Univ. Press, 1991, p. 169, avec la bibliographie.
}

9 NAGY, Pindar's Homer; cit. (n. 5), p. 216. 
Pythagore et des Puthagoreioi, des «Pythagoriciens ${ }^{10}$. Si Orphée et Musée sont d'origine égyptienne, alors, par défaut, Homère et Hésiode peuvent devenir, pour Hérodote, les plus anciens poètes des Grecs. Pourtant, la conception traditionnelle $\mathrm{du} \mathrm{v}^{\mathrm{e}}$-Iv $\mathrm{I}^{\mathrm{e}}$ siècle semble avoir placé Orphée et Musée avant Homère et Hésiode, sans leur donner une origine égyptienne.

'C'est ici qu'il devient extrêmement important de considérer les versions pergaméniennes des métaphores de «cosmos » et de "corpus " autour du texte homérique, puisque leur source semble être liée à la figure d'Orphée, mais indépendamment d'une origine égyptienne.

Commençons par la métaphore du " cosmos », qui relève de la méthodologie de Cratès comme éditeur d'Homère. L'édition d'Homère par Cratès de Pergame et celle d'Aristarque d'Alexandrie sont radicalement différentes, et les différences peuvent être saisies dans l'instantané de l'exemple suivant:

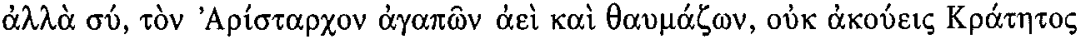
$\dot{\alpha} \nu \alpha \gamma i v \omega ́ \sigma \kappa o v \tau o \varsigma$

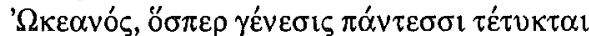

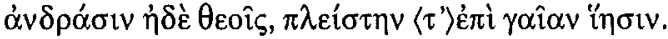

Mais toi, qui n'as de goût et d'admiration que pour Aristarque, tu n'écoutes (akouô) pas Cratès lire ces vers :

L'Océan, chez qui tous ont trouvé la naissance

Les hommes et les dieux, couvre presque la terre

(Plut., De facie in orbe lunae, $983 \mathrm{~d}$ [trad. d'après P. Raingeard, Paris, 1935])

Le premier des deux vers cités par Plutarque correspond au vers 246 du chant XIV de l'Iliade (avec le début du vers ' $\Omega \kappa \varepsilon \alpha v o ́ \varsigma$ poursuivant la syntaxe du vers 245 tel qu'il nous est conservé), tandis que le deuxième (XIV, 246a) a été éliminé du texte des éditions modernes standards de l'Iliade ${ }^{11}$.

Pour Cratès (fr. 32-33 Mette), ce vers additionnel soutient la théorie cosmique selon laquelle Okéanos était la mer salée couvrant la Terre conçue comme sphérique. Cette théorie était réfutée par Aristarque qui prétendait que l'Okéanos homérique devait être vu comme un fleuve d'eau douce entourant une Terre ronde et plate. Ce qui était alors en jeu, du moins dans le cas de Cratès et d'Aristarque, ne relevait pas de préoccupations insignifiantes. En cette matière, les enjeux étaient en fait de dimension cosmique. Et il est sans doute ironique d'écrire cela en un temps où la mode est au rejet des

10 Voir M.L. WEst, The Orphic Poems, Oxford, Clarendon Press, 1983, p. 8 : pour Hérodote, «orphique » et «bacchique » peuvent en réalité être des équivalents de « égyptien » et « pythagoricien » (e.g. II, 81).

11 Par exemple, XIV, $246 a$ est relégué dans l'apparat critique de l'Oxford Classical Text de D.B. Munro et Th. W. Allen (Oxford, Univ. Press, 1920 ${ }^{3}$ ). Il est totalement absent de l'édition de l'lliade par H. van Thiel (Hildesheim, Olms, 1996). 
variantes textuelles homériques sous prétexte qu'elles sont « futiles », «banales » ou même « ennuyeuses ».

Il est plus ironique encore de voir que les éditions modernes d'Homère ignorent les variantes textuelles signalées et interprétées par Cratès. La recherche actuelle aurait bien pu distinguer Cratès pour ses intuitions scientifiques envisageant une terre sphérique plutôt que circulaire, s'il n'avait pas fondé son raisonnement sur le texte d'Homère. Il nous est très difficile d'appréhender une période de l'histoire intellectuelle lorsque le prestige de toute étude de haut niveau est centré sur l'étude d'Homère. L'association moderne de Cratès avec la critique homérique est allée jusqu'à en minimiser le statut potentiel de critique littéraire, alors que les preuves sont suffisantes, parmi le peu qu'il reste de la critique homérique de Cratès, pour acclamer en lui un critique littéraire très fin et sensible, un critique dont les interprétations égalent, sinon même surpassent, celle du pseudo-Longin dans son Traité $d u$ sublime.

Même du point de vue de la critique homérique, les choix éditoriaux de Cratès reflètent un solide bagage de preuves textuelles. On pourra souvent choisir de rejeter des points particuliers d'interprétation, mais les variantes textuelles qu'il ajoute ne peuvent être discréditées comme relevant de l'invention pure et simple. Par exemple, il ressort d'une analyse empirique de la composition formulaire du vers 246 a du chant XIV de l'Iliade qu'il n'y a rien qui ne soit traditionnel dans la forme du vers ajouté par Cratès, pas plus que dans le contenu. De plus, d'un point de vue formulaire, le vers n'induit pas nécessairement une vision de mer salée, sans parler d'une terre sphérique, comme l'argumente Cratès. C'est dans le contexte immédiat d'Okéanos que le vers diffère d'autres vers. Si nous suivons la séquence des vers 246246a, l'idée qui s'en dégage est qu'Okéanos n'engendre pas «tout » - ce qui serait le thème exprimé par le vers 246 sans le vers $246 \mathrm{a}$ - mais, plus précisément, tous les dieux et les hommes. Cette idée-là non plus n'a rien qui ne soit traditionnel. En effet, il paraît tout aussi archaïque, dans une perspective conventionnelle, de dépeindre Okéanos comme un père anthropomorphe des dieux et des hommes qu'en tant que géniteur cosmique de l'univers.

Nous pouvons noter avec intérêt l'orientation traditionnelle des vers 246$246 \mathrm{a}$ du chant XIV de l'Iliade tels que les lit Cratès : c'est décidément " orphique », reflétant les traditions poétiques et interprétatives attribuées par les anciens à Orphée ${ }^{12}$. Je pense que Cratès a puisé ces vers à un « Homerus auctus », une tradition homérique augmentée de traditions « orphiques ». Je fais même l'hypothèse de l'existence de «vers ajoutés » émanant d'éditions d'Homère contaminées par ces traditions orphiques.

12 Voir H. HELCK, De Cratetis Mallotae studiis criticis quae ad Iliadem spectant, Leipzig, Noske, 1905, p. 30-31. 
Du point de vue des érudits de Pergame, toutefois, de tels ajouts « orphiques » dans la tradition textuelle homérique n'étaient pas du tout des « contaminations », puisqu'Orphée aussi bien que Musée avaient, supposaiton, vécu avant Homère ${ }^{13}$. La pratique éditoriale pergaménienne incluait manifestement des vers « orphiques», qui étaient ajoutés au texte homérique, alors que la pratique alexandrine les excluait, c'est-à-dire qu'elle les omettait purement et simplement du texte au lieu de les athétiser. Une telle différence dans les pratiques éditoriales semble attestée par le texte suivant, qui conserve le souvenir d'un bon mot antique :

annales evolvam omnium gentium et quis primus carmina scripserit quaeram? Quantum temporis inter Orpbea intersit et Homerum, cum fastos non babeam, computabo? Et Aristarcbi ineptias, quibus aliena carmina conpunxit, recognoscam et aetatem in syllabis conteram?

Vais-je compulser l'histoire de toutes les nations pour savoir qui le premier a fait des vers? Vais-je supputer, faute de fastes pour me documenter, le laps de temps qui sépare Orphée d'Homère? Vais-je recenser les notes pédantesques dont Aristarque lardait (conpunxit) les poèmes d'autrui et user ma vie sur des syllabes ? $^{14}$

(Sénèque, Lettres, 88,39 [trad. d'après H. Noblot, CUF, 1995])

Ici, le thème de l'absence des témoignages écrits (fasti) d'Orphée et d'Homère reflète ce qui semble avoir été un désaccord sans fin entre les sectateurs de Cratès et ceux d'Aristarque quant à la provenance d'Homère.

Aristarque, pour sa part, croyait qu'Homère était un Athénien vivant autour de 1000 av. J.-C. (scholie A à l'lliade XIII, 197), et qu'il avait consigné par écrit l'Iliade et l'Odyssée (scholie A à l'Iliade XVII, 719) ${ }^{15}$. Par contre, il n'existe pas d'indication que Cratès ait considéré Homère comme un Athénien, sans parler de la mise par écrit de ses propres poèmes ${ }^{16}$. Au contraire, Cratès semble avoir suivi la tradition narrative de la « transcription pisistratique » selon laquelle de fortes lacunes apparaissaient dans la tradition écrite d'Homère jusqu'au moment où les Pisistratides rassemblèrent le « corpus ».

13 Voir NagY, Pindar's Homer, cit. (n. 5), p. 216, n. 10.

14 Je remercie Jed Wyrick qui m’écrit (dans une lettre datée du 15 février 1998) : “I think that there is a clear contrast here between two different kinds of "absurd" approaches to learning - the one involving Orpheus, and the other, Aristarchus, »

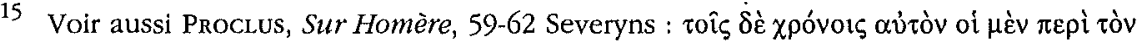

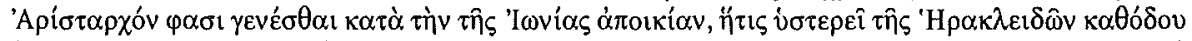

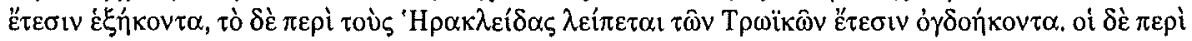

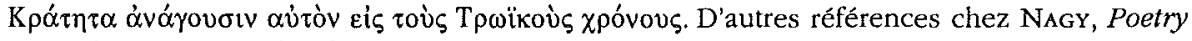
as Performance, cit. (n. 8), p. 151.

16 Helck, o.c. (n. 12), p. 63 et 74-75: Cratès ne considérait pas Homère comme un Athénien, bien qu'il puisse avoir pensé qu'Homère utilisait des atticismes à l'occasion. 
Je veux démontrer que la tradition narrative de la «transcription pisistratique » est déterminée par la seconde des deux métaphores touchant à l'édition du texte homérique, celle de «corpus». En termes de tradition, le corpus de la poésie homérique était démembré et éclaté, jusqu'à l'intervention, soit de Pisistrate, soit de son fils, selon certaines versions, ou de Solon, selon d'autres - qui réunit des gens pour recomposer le corpus démembré ${ }^{17}$.

La scholie $\mathrm{H}$ au vers 604 du livre XI de l'Odyssée rapporte que ce vers homérique (ou le passage entier : XI, 602-604) était l'œuvre d'Onomacrite. D'après Tzetzès (Sur la Comédie, p. 20 Kaibel), Onomacrite était l'un des quatre rédacteurs responsables de la transcription pisistratique ${ }^{18}$. Ailleurs, comme chez Pausanias (IX, 35, 5), Onomacrite était le pseudonyme d'un auteur de poèmes « orphiques ${ }^{19}$. En général, la forme finale des poèmes « orphiques » est « unmistakably connected with the Pergamene account of the Pisistratean recension of the Homeric poems ${ }^{20}$. La division du corpus orphique en 24 rbapsôidiai, "rhapsodies ", pourrait être une indication de cette relation ${ }^{21}$.

On a prétendu que l'« inventeur » de l'idée même d'une transcription pisistratique était un érudit pergaménien, Asclépiade de Myrlea (c. 100 av. J.C.), dont le but avait été de fournir un contrepoids aux théories alexandrines sur l'origine d'Homère. Au contraire, j'ai argumenté en faveur d'une datation plus haute pour cette mise au point, plus ou moins contemporaine de l'époque des Pisistratides eux-mêmes. À l'appui de cette argumentation, nous pouvons noter qu'Onomacrite est déjà mentionné par Hérodote (VII, 6, 3) comme collaborateur des Pisistratides, au titre de diathetês, " arrangeur », de la poésie oraculaire à des fins politiques. Dans ce contexte, Onomacrite est en outre accusé par Hérodote d'avoir fait des ajouts frauduleux à la poésie oraculaire de Musée. De telles additions auraient dû être athétisées par les érudits de Pergame, bien que l'on s'attende à trouver ces atbeta maintenus dans le texte.

17 Discussion détaillée dans G. NAGY, Homeric Questions, Austin, Univ. of Texas Press, 1996, p. 70-75, 93-112.

18 WEST, o.c. (n. 10), p. 249.

19 Ibid., p. 221, n. 141 et p. 250 , n. 43.

20 Ibid., p. 249.

21 Ibid., p. 248 : il pense que le compilateur était un certain Theognetos, mentionné par la Souda, qui divisa son ceuvre en 24 rouleaux (p. 232-233). Dans Orpbica F 157, le sceptre qui passe à Zeus (six rois en tout, et il est le cinquième) comptait 24 mesures. WEST (p. 232) fait la comparaison avec Orpbica F 356: "droit, en six parties, de 24 mesures ». Une telle formule, attribuée tantôt à Orphée, à Musée ou à la Pythie, renvoie à l'hexamètre, qui contient six pieds et 24 parties. Comme WEST le remarque (p. 232), ces trois mêmes " auteurs " sont les différents " inventeurs " traditionnels de l'hexamètre. De même dans l'Ulysse attribué à Alkidamas, Musée est l'« inventeur " de l'hexamètre, comme chez Démocrite (68 B 15 D-K). J'ajouterais, toutefois, que la division de la théogonie orphique en 24 rhapsodies n'est pas nécessairement modelée sur le schéma homérique, comme le veut WEST (p. 249). 
Du fait qu'Aristarque a ouvert, dans ses propres publications, un débat très large avec Cratès sur la critique textuelle d'Homère, il me semble plus vraisemblable de considérer la construction par Aristarque d'un Homère athénien qui aurait écrit ses poèmes autour de l'an 1000 av. J.-C. comme un contrepoids à l'idée d'une transcription pisistratique acceptée par Cratès et l'école de Pergame ${ }^{22}$. Asclépiade de Myrlea est simplement l'un des représentants tardifs les plus visibles de cette conception plus ancienne ${ }^{23}$.

En fait, si la construction par Aristarque d'un Homère athénien est une innovation conçue pour aller à l'encontre de l'idée plus ancienne de la transcription pisistratique, telle que l'acceptaient Cratès et l'école de Pergame, nous sommes mieux à même de comprendre le raisonnement de Flavius Josèphe lorsqu'il affirme que les chants d'Homère ne furent pas mis par écrit mais remémorés au fil du temps, puis éclatés et finalement rassemblés :

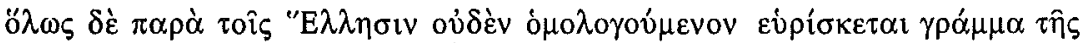

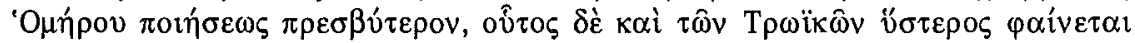

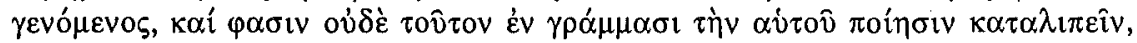





Nulle part, d'ailleurs, en Grèce, on ne trouve un écrit reconnu plus ancien que la poésie d'Homère. Or, il est clair que ce poète est encore postérieur à la guerre de Troie. Et lui-même, dit-on, ne laissa pas ses poèmes par écrit; mais, transmis par la mémoire (diamnêmoneuomenên), ils furent plus tard constitués (suntethênai) par la réunion des chants; de là, les nombreuses divergences qu'on y constate.

(Flavius Josèphe, Contre Apion I, 12 [trad. L. Blum, CUF, 1930l)

À l'évidence, Flavius Josèphe utilisait l'ancienne hypothèse de la transcription pisistratique pour miner l'idéologie d'Apion, son opposant aristarquéen.

Selon le modèle de la succession Orphée-Homère, telle que l'acceptait l'école pergaménienne de Cratès, le texte de l'« Homerus auctus » inclut des éléments orphiques. Selon le modèle de la succession Homère-Orphée, telle que l'acceptait l'école alexandrine d'Aristarque, le texte d'Homère implique le rejet des éléments orphiques.

22 Cette explication renverse les orientations du scénario mis en place par WEST (p. 249) : «To counter Aristarchus' arguments for an Athenian Homer, the theory was developed that the rbapsôidiai, "récitations", into which the Homeric poems were divided, represented episodes which Homer had recited and left behind him in different towns; they had then been united by Pisistratus with the help of certain poets, who recreated an approximation to Homer's original conceptions, but interpolated passages of their own, which accounted for Attic elements. "

23 Asclépiade est cité comme l'autorité qui associa Orphée de Crotone à Pisistrate : Souda, s.v. Orpheus Krotôniatês $=697$ F 9 J. Voir WEst, o.c. (n. 10), p. 249-250, sur Orphée de Crotone comme « homonym split » (d'autres exemples p. 250, n. 41). 
Selon le modèle pergaménien de la succession Orphée-Homère, l'Iliade et l'Odyssée homériques comptent 24 rhapsôidiai parce qu' "Orphée » comptait déjà 24 rhapsôidiai. Cette tradition est ionienne et articulée autour des 24 lettres de l'alphabet ionien.

Ainsi, les Homerica et Orphica de Pergame étaient spéculativement issus d'une " transcription pisistratique » des deux corpus; d'où l'association d'un personnage comme Onomactite à la fois avec les Homerica et les Orphica. En termes de "transcription pisistratique » comme "mythe de fondation ", l'« origine » des Homerica et des Orpbica devait être ionienne et non attique. La métaphore du «corpus » appliquée aux Homerica et aux Orpbica est analogue au thème tradtionnel du démembrement explicite d'Orphée et de son remembrement implicite (mystique). La métaphore du « cosmos» appliquée aux Homerica et aux Orpbica est analogue au thème traditionnel d'Orphée chantre de cosmogonies.

Harvard University

Gregory NAGY

Department of Classics

Boylston Second Floor

CAMBRIDGe MA 02138 